\title{
Monetary and non-monetary rewards reduce attentional capture by emotional distractors.
}

\author{
Amy T. Walsh ${ }^{1,2}$, David Carmel ${ }^{1,3}$, David Harper ${ }^{1}$, Petra Bolitho ${ }^{1}$, and Gina M. \\ Grimshaw ${ }^{1}$
}

${ }^{1}$ School of Psychology, Victoria University of Wellington, New Zealand

${ }^{2}$ Department of Clinical Neuroscience, Division of Psychology, Karolinska Institutet, Stockholm, Sweden

${ }^{3}$ Department of Psychology, University of Edinburgh, UK

Address Correspondence to: Amy Walsh ${ }^{1}$, School of Psychology, Victoria University of Wellington, PO Box 600, Wellington 6140, New Zealand; walsh.amy.t@gmail.com; ORCiD 0000-0001-69725550; twitter @walsh_aims. Other authors: Gina Grimshaw, School of Psychology, Victoria University of Wellington, PO Box 600,Wellington 6140, New Zealand. gina.grimshaw @vuw.ac.nz; ORCiD 0000-0002-1291-1368; twitter @ ginagrimshaw. David Carmel², School of Psychology, Victoria University of Wellington, PO Box 600, Wellington 6140, New Zealand; david.carmel@vuw.ac.nz; ORCiD 0000-0003-1874-158X. David Harper, School of Psychology, Victoria University of Wellington, PO Box 600, Wellington 6140, New Zealand, david.harper@vuw.ac.nz. Petra Bolitho, School of Psychology, Victoria University of Wellington, PO Box 600, Wellington 6140, New Zealand, petrabolitho@ gmail.com, ORCiD 0000-0001-8088-4798.

Materials, data and analyses are available at https://osf.io/pm4wj/

Word count including abstract and references: 8116

\footnotetext{
${ }^{1}$ Other affiliations: Department of Clinical Neuroscience, Division of Psychology, and Department of Neurobiology, Care Sciences and Society, Aging Research Centre, Karolinska Institutet, Stockholm, Sweden. 2 Psychology Department, University of Edinburgh, 7 George Square, Edinburgh EH8 9JZ, UK
} 


\title{
Monetary and non-monetary rewards reduce attentional capture by emotional distractors.
}

\author{
Irrelevant emotional stimuli often capture attention, disrupting ongoing cognitive processes. \\ In two experiments, we examined whether availability of rewards (monetary and non- \\ monetary) can prevent this attentional capture. Participants completed a central letter \\ identification task while attempting to ignore negative, positive, and neutral distractor images \\ that appeared above or below the targets on $25 \%$ of trials. Distraction was indexed by slowing \\ on distractor-present trials. Half the participants completed the task with no performance- \\ contingent reward, while the other half earned points for fast and accurate performance. In \\ Experiment 1, points translated into monetary reward, but in Experiment 2, points had no \\ monetary value. In both experiments, reward reduced capture by emotional distractors, \\ showing that even non-monetary reward can aid attentional control. These findings suggest \\ that motivation encourages use of effective cognitive control mechanisms that effectively \\ prevent attentional capture, even when distractors are emotional.
}

Keywords: motivation; reward; cognitive control; emotion; distraction 
At any moment, a multitude of sensory signals compete for awareness (Desimone \& Duncan, 1995). Stimuli that elicit emotions are particularly effective at capturing peoples' attention (Dolcos et al., 2019; Okon-Singer, Lichtenstein-Vidne, \& Cohen, 2013; Pourtois, Schettino, \& Vuilleumier, 2013; Vuilleumier, 2015; Yiend, 2010). These emotional stimuli are often relevant to biologically-determined survival goals, and therefore usually guide behaviour adaptively (Lang \& Bradley, 2010; LeDoux, 2012; Rolls, 2000). But emotional stimuli can also distract people from achieving more mundane daily goals (Grimshaw, Kranz, Carmel, Moody, \& Devue, 2018; Gupta, Hur, \& Lavie, 2016; Most, Chun, Widders, \& Zald, 2005; Okon-Singer, Tzelgov, \& Henik, 2007; Padmala \& Pessoa, 2014): social media notifications divert attention from work; temptations like wine and chocolate undermine healthy choices; anxious thoughts make it difficult to focus. Can motivation act as a shield against such distractions?

The ability to focus on goal-relevant information while filtering out irrelevant distractions depends on the use of cognitive control (Cohen, 2017; Gratton, Cooper, Fabiani, Carter, \& Karayanidis, 2018; Miller, 2000). According to the Dual Mechanisms of Control (DMC) framework (Braver, 2012; Braver, Gray, \& Burgess, 2007), people tend to flexibly shift between two control strategies - each comprising a constellation of mechanisms depending on the context. Reactive control mechanisms detect and resolve distraction after it has occurred, returning attention to the goal; in contrast, proactive control mechanisms anticipate and prevent distraction before it can occur. A key premise of the DMC framework is that proactive control is a more effective shield against distraction, but requires greater effort. A shift to proactive control therefore occurs only when the perceived benefits outweigh the costs (Botvinick \& Braver, 2015; Kool, Shenhav, \& Botvinick, 2017; Shenhav, 2017). 
As predicted by the DMC framework, high task motivation encourages a shift to proactive control in non-emotional contexts (Botvinick \& Braver, 2015; Chiew \& Braver, 2014; Etzel, Cole, Zacks, Kay, \& Braver, 2016; Padmala \& Pessoa, 2011; van den Berg, Krebs, Lorist, \& Woldorff, 2014; Yee \& Braver, 2018). Less is known, however, about how motivation influences control when distractors are emotional. Biased attention to emotional stimuli occurs via both salience-driven (Carretié, 2014; Mulckhuyse, 2018; Öhman, Flykt, \& Esteves, 2001) and goal-driven mechanisms (Brown, Berggren, \& Forster, 2019; Mohanty \& Sussman, 2013), and there may even be specific neural pathways dedicated to emotional attention (Pourtois et al., 2013; Vuilleumier, 2015). Moreover, responding to emotional stimuli is often crucial for survival (LeDoux, 2012), and so ignoring unexpected emotional stimuli could be dangerous or costly. Given the special status of emotional stimuli, emotional distractions may be filtered via different control mechanisms than non-emotional ones. The same mechanisms that encourage proactive control in non-emotional contexts may therefore be ineffective against emotional distractions.

Despite these strong perceptual biases, empirical evidence shows that people can sometimes ignore emotional distractors. For example, emotional distraction can be reduced or eliminated by difficult tasks that consume attentional resources (e.g., Gupta et al., 2016; Okon-Singer et al., 2007), or by presenting distractors frequently (e.g., Grimshaw et al., 2018; Schmidts, Foerster, Kleinsorge, \& Kunde, 2020). A small but growing body of evidence shows that increasing task motivation can also reduce emotional distraction, even by high arousal images (Padmala \& Pessoa, 2014; Padmala, Sirbu, \& Pessoa, 2017; Walsh, Carmel, \& Grimshaw, 2019; Walsh, Carmel, Harper, \& Grimshaw, 2018). Motivation is typically manipulated by availability of points that are contingent on fast and accurate performance. The points then translate into greater monetary reward upon task completion. For example, in two studies, Padmala and colleagues (Padmala \& Pessoa, 2014; Padmala et 
al., 2017) had participants judge the orientation of lines on either side of a central, taskirrelevant image that could be either negative or neutral. Cues indicated whether reward was available at the beginning of each trial. On unrewarded trials, responses were slower on negative relative to neutral distractor trials, but this difference was eliminated on rewarded trials. Similarly, in two studies (Walsh et al., 2018; 2019), we had participants identify a target letter that appeared above or below a central task-irrelevant image that could be positive, negative, or neutral. Half of the participants completed the task with no reward; the other half could earn a reward on every trial for fast and accurate performance. Performance was slower on positive and negative, relative to neutral, distractor trials. But rewarded participants showed less emotional distraction. Findings extend the DMC framework to emotional contexts, suggesting that proactive control can effectively guard against potent emotional distractions.

In the four studies described thus far, participants responded to peripheral target stimuli that appeared above, below, or to either side of, central fixated distractors (Padmala \& Pessoa, 2014; Padmala et al., 2017; Walsh et al., 2018; 2019). But in real life, distractors often capture attention from outside our current gaze location. Foveal vision (at fixation) is known to have greater spatial resolution (Berardi \& Fiorentini, 1991), but lower temporal resolution (Carrasco, McElree, Denisova, \& Giordano, 2003) than peripheral vision, and the two are supported by at least partly separable neural substrates (Azzopardi \& Cowey, 1993). Although it is possible to covertly attend to locations outside of fixation (Posner, 1980), attention is tightly linked to foveal vision (Corbetta et al., 1998). Greater attention is allocated to central than peripheral distractors, as indicated by both behavioural (Beck \& Lavie, 2005) and neural (Carretie et al., 2013) markers. Because attention functions differently in peripheral versus central locations, it is possible that attentional control might also function differently for central versus peripheral distractors. Thus, the known effects of motivation on 
the processing of fixated distractors cannot be assumed to generalize to peripheral distraction. Our primary aim here is to extend previous findings by determining whether motivation can reduce attentional capture by peripheral emotional distractors.

In two experiments, we use an adapted version of the irrelevant distractor paradigm (Forster and Lavie, 2008). Participants were instructed to ignore positive, negative, and neutral distractors that were presented on $25 \%$ of trials, either above or below central letter targets. Participants were randomly allocated to perform the distraction task with (reward group) or without (control group) performance-contingent reward. In Experiment 1, participants were rewarded with points that then translated into monetary earnings. In Experiment 2, we determined whether motivation can be elicited by non-monetary reward by awarding points that were purely symbolic and had no monetary value.

\section{Experiment 1}

We expected emotional images to be more distracting than neutral images, and for reward to speed responses overall. Our main question, though, was whether motivation enhances cognitive control to reduce attentional capture by emotional distractors. If so, we should find less distraction in the reward group than the control group. Moreover, if the mechanisms that control emotional distraction by peripheral images are similar to those that control emotional distraction by central images, reward should reduce emotional distraction to a greater extent than neutral distraction, as found previously for central, fixated distractors (Walsh et al., 2018; 2019).

\section{Method}

\section{Participants and Sample Size Determination}

Sixty-six participants were recruited from the community in Wellington, New Zealand, via flyers, emails, and social media. All participants were women, to reduce within-group variance, and to allow for use of images that were calibrated for valence and arousal ratings 
that differ across genders. Following our standard laboratory procedures, one participant was excluded for achieving less than $75 \%$ accuracy overall and achieving less than $60 \%$ accuracy on a single block. Another participant was excluded due to a technical error during data collection. The final sample included 64 participants $(M=23$; range 18-39 years), who were randomly assigned to the control and reward groups ( $n=32$ per group). The effect size of reward on emotional distraction in Walsh et al. (2018) was $d_{s}=0.519$; and in Walsh, et al. (2019) was $d_{s}=0.939$. We used the average of those effect sizes $\left(d_{s}=0.729\right)$ as the benchmark in a sensitivity analysis using G*Power (Faul, Erdfelder, Lang, \& Buchner, 2007), which indicated that a sample size of 32 participants per group had a 0.8 probability of detecting an effect of reward on distraction greater than $d_{s}=0.711$ (alpha $=.05$, two-tailed). Control participants received a $\$ 15$ supermarket voucher for participating. Reward participants understood they could earn between $\$ 10$ and $\$ 20$, contingent on performance.

Participants in both experiments spoke fluent English, had normal or corrected-tonormal vision, and reported no current treatment for depression or anxiety. Both experiments were approved by the Victoria University of Wellington Human Ethics Committee, and participants gave informed consent before participating. Analyses were undertaken after all data were collected.

\section{Materials}

Participants were run in groups of one to four (all in the same experimental condition) in a dimly-lit room, seated in separate booths. The experiment was run using E-Prime 2.0 (Psychology Software Tools, Pittsburgh, PA, USA), on Dell Precision T1700 personal computers with 24 " monitors at $1920 \times 1080$-pixel resolution and a $120 \mathrm{~Hz}$ refresh rate. A chin-rest maintained a viewing distance of $57 \mathrm{~cm}$. 
Twelve images for each category (negative, neutral, positive) were drawn from the International Affective Picture System (IAPS, Lang et al., 2008; see Table S.1 for mean arousal and valence ratings). The positive images (scenes of heterosexual erotic couples) and negative images (scenes of body mutilations) were matched on arousal using normative ratings from women (Lang et al., 2008). The neutral images contained people and therefore had biological and social relevance, but lacked emotional valence, and were rated low in arousal. All images were matched for luminance using the SHINE MATLAB toolbox (Willenbockel et al., 2010).

Participants also completed the Behavioural Activation and Inhibition Scale (BIS/BAS; (Carver \& White, 1994). Scores on the BAS-drive subscale were used for exploratory analyses to inform the design of future experiments on the influence of individual differences in reward sensitivity on performance and are not given further consideration here.

\section{Task and Procedure}

Figure 1 illustrates the distraction task. For all participants (regardless of reward condition) each trial began with a central white fixation cross (jittered duration between 417-833 ms) presented on a black background, followed by a $100 \mathrm{~ms}$ target display (short enough to preclude saccades to the distractors; Walker, Walker, Husain, \& Kennard, 2000), of six white letters arranged in a circle around fixation (radius $1.75^{\circ}$ ). Each display included a target letter $\left(\mathrm{K}\right.$ or $\left.\mathrm{N} ; 0.67^{\circ} \times 0.36^{\circ}\right)$, the other five letters were o's $\left(0.22^{\circ} \times 0.22^{\circ}\right)$. On $25 \%$ of trials, a distractor image $\left(6.68^{\circ} \times 6.68^{\circ}\right.$, centred at $6.68^{\circ}$ eccentricity $)$ was presented simultaneously above or below the letter display. Participants were instructed to ignore the images, and to respond as quickly and accurately as possible, with their dominant hand, to indicate whether $\mathrm{K}$ or $\mathrm{N}$ was present using keys 1 and 2 (counterbalanced across participants) on the numberpad . Participants responded during a response-window of $1900 \mathrm{~ms}$ from stimulus-offset. Failure to respond was recorded as an error. Response was followed by a $600 \mathrm{~ms}$ blank 
screen and then visual feedback (100 ms duration): "correct" in green, "incorrect" in red, or "please respond faster" in white (if no response was made within the response-window). A random inter-trial-interval ranged between 207-623 ms. Target letter and location were counterbalanced across trials, and trial order was randomised within blocks.

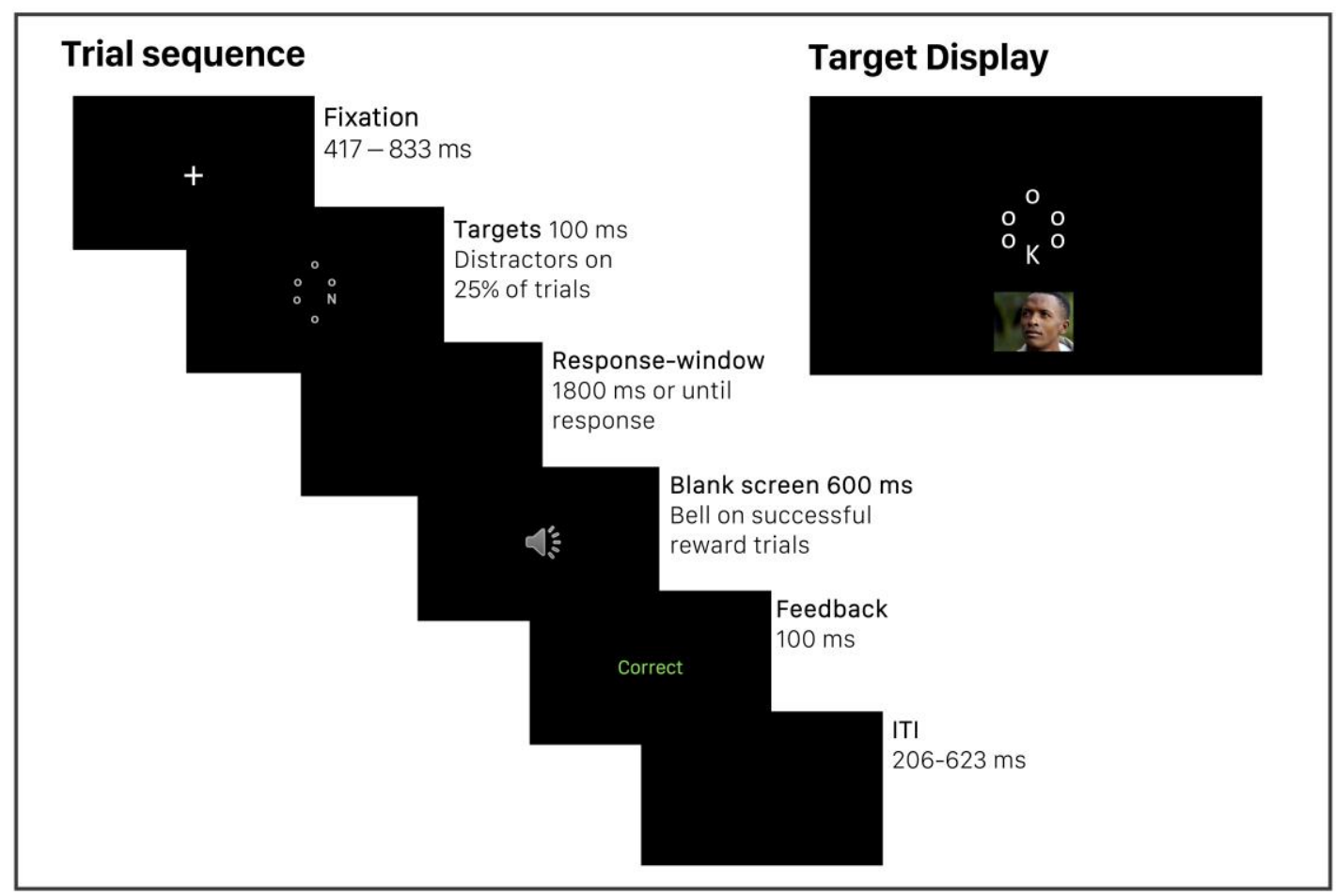

Figure 1. Trial sequence (left) for participants in the reward group, and the target display (right) for the distraction task. The trial sequence (left) depicts a successful reward trial, with no distractor. The target display (right) depicts the stimuli of a neutral distractor-present trial. Distractors were presented on $25 \%$ of trials, and were blocked by valence (negative, neutral, positive). Participants in the control condition experienced the same trial sequence, but with no bell sound. Images are not to scale; the central part of the display is enlarged for illustrative purposes.

Participants completed 24 practice trials (six distractor-present, 18 distractor-absent) to become familiar with the task, and a baseline block of 48 trials (12 distractor-present, 36 distractor-absent) to establish their median RT, which served as the criterion for reward in the rest of the experiment. The practice and baseline blocks contained scrambled, instead of intact, images as distractors (created from different images than those presented on 
experimental trials). To create scrambles, images were divided into $36 \times 36$ segments that were randomly recombined in PhotoScape-v3.7.

To limit valence carry-over effects, the experiment was split into two halves of 192 trials each (counterbalanced across participants), separated by a 3-minute break. Each half comprised two emotional (positive or negative) and two neutral blocks, presented in ABBA order and separated by self-timed breaks. This yielded four block orders, which were counterbalanced across participants. Within a block (48 trials; 12 distractor-present, 36 distractor-absent), all images were either negative, neutral, or positive, randomly mixed with distractor-absent trials. Each image was presented once in a block, although images were repeated (emotional images twice; neutral images four times) across the experiment to increase trial numbers to obtain stable measures of response times (RTs). Experiment duration was approximately 50 minutes.

\section{Reward Condition}

After the baseline block, reward participants were informed about the potential to earn one point per trial if they were correct and faster than their median RT from the baseline block. Success was indicated by a pleasant sound (600 ms duration) immediately following response. Participants began on Level 1 (\$10) and advanced a level with every 91 points earned; each level increased earnings by $\$ 2.50$, until a maximum was reached at Level 5 (\$20). During the breaks between blocks, points total and current level appeared above an animated display of falling coins, along with mean RT and percent accuracy for the preceding block. Participants were encouraged to be fast and accurate to get to the next level. A warning was provided encouraging people to keep accuracy high if it fell below $95 \%$ in a block. Reward participants all earned between $\$ 15$ and $\$ 20(M=\$ 16.41)$.

\section{Control Condition}


Control participants were not aware of the reward condition. They were given $\$ 15$ in return for participation, which was not contingent on performance. They received no auditory feedback on a trial-by-trial basis. During the breaks, instead of performance feedback and the coin animation, they saw 'please wait...' and instructions to take a break. In all other respects they completed the same task as the reward participants.

\section{Analyses}

RTs faster than $200 \mathrm{~ms}$ were excluded as anticipatory ( $<0.01 \%$ of trials in each experiment). A high cut-off was not used as the response-window was limited to $2000 \mathrm{~ms}$ from stimulusonset. All reported effect sizes for Analyses of Variance (ANOVAs) are $\eta_{p}{ }^{2}$ Lakens, 2013). As recommended by Delacre, Lakens, and Leys (2017), Welch's tests are used as the default (instead of independent $t$-tests) for between-subjects comparisons as they are robust to violations of heterogeneity of variance. Effect sizes are Cohen's $d_{s}$ for between-subject comparisons, and Cohen's $d_{z}$ for within-subject comparisons (Cohen, 1992; Lakens, 2013). Degrees of freedom are adjusted for violations of sphericity (Greenhouse-Geisser correction) when necessary. Effects are considered significant if $p<.05$. Analyses were performed in Jamovi (2019). Figures were produced in RStudio (2019) using ggplot2 (Wickham, 2016). Data and code to reproduce analyses and figures are available at https://osf.io/pm4wj/.

\section{Results and Discussion}

Mean correct RTs and accuracy for neutral distractor blocks did not differ across the positive and negative halves of the experiment (distractor-present and distractor-absent conditions; all $p$ 's > .361), so we collapsed the neutral distractor blocks in analyses to create three valence conditions (negative, neutral, positive). Because our hypotheses predicted effects of reward on distraction, we also calculated RT and accuracy distraction indices as the difference between distractor-present and distractor-absent trials in the same block. Mean correct RTs, RT distraction indices [distractor-present RT - distractor-absent RT], mean proportion correct 
scores, and accuracy distraction indices [distractor-absent proportion correct - distractorpresent proportion correct] for each condition are presented in Table 1. RT distraction indices are displayed in the top panel of Figure 2. To control for possible differences in overall RT across reward conditions, we also created a proportional RT distraction index [distractorpresent RT - distractor-absent RT / distractor-absent RT * 100] that reflects percentage slowing of responses on distractor-present trials, relative to distractor-absent trials. Analyses of proportional distraction indices are in Supplementary Materials. 

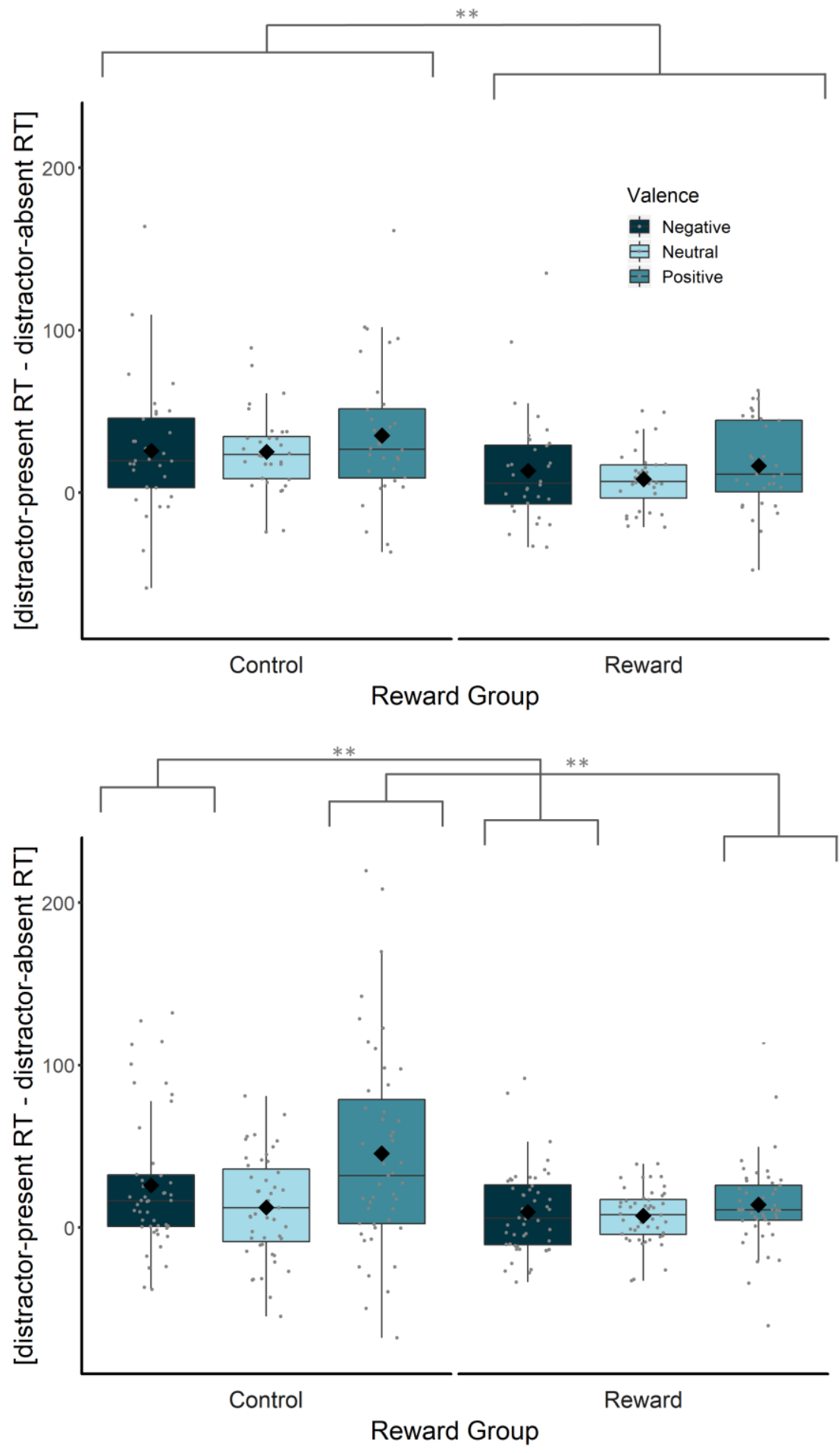

Figure 2. Mean behavioural distraction indices (in ms) by reward group and valence for Experiment 1 (top panel) and Experiment 2 (bottom panel). In Experiment 1, distraction (overall) was attenuated by reward. In Experiment 2, positive and negative emotional distraction, but not neutral distraction, were attenuated by reward. Individual dots indicate individual participants. Diamonds represent the mean of their condition. The horizontal line indicates the median for that condition. The upper and lower edges of the boxes reflect the first and third quartiles. The top and bottom ends of the whiskers reflect the largest and smallest values no further than 1.5 times the interquartile range. 
Table 1. Experiment 1. Mean correct RTs (ms), mean accuracy (proportion correct), RT distraction indices [distractor-present RT - distractor-absent RT], and accuracy distraction indices [distractor-absent RT - distractor-present RT] for each distractor condition (negative, neutral, positive, and distractor-absent), are shown separately for control and reward groups. SDs are in brackets.

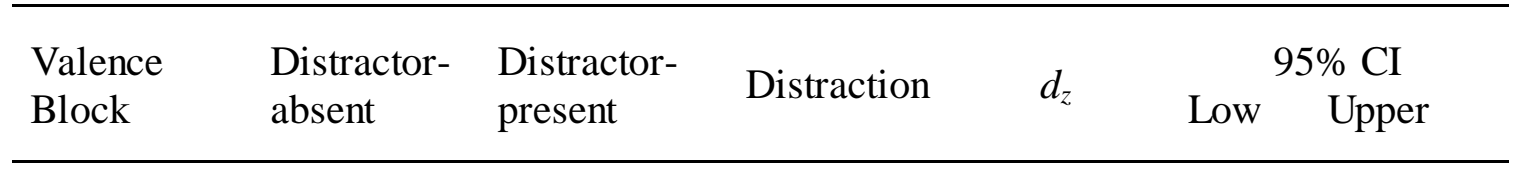

$\underline{\text { Response Times }}$

Control Group

\begin{tabular}{|c|c|c|c|c|c|c|}
\hline Negative & $526(59)$ & $555(82)$ & $29(36) * * *$ & 0.809 & 16 & 42 \\
\hline Neutral & $531(60)$ & $555(74)$ & $24(25) * * *$ & 0.958 & 15 & 33 \\
\hline Positive & $532(59)$ & $565(79)$ & $33(38) * * *$ & 0.861 & 19 & 47 \\
\hline \multicolumn{7}{|c|}{$\underline{\text { Reward Group }}$} \\
\hline Negative & $472(50)$ & $486(68)$ & $14(29) * *$ & 0.500 & 4 & \\
\hline Neutral & $474(55)$ & $481(54)$ & 7 (22) & 0.314 & -1 & \\
\hline Positive & $473(54)$ & $489(64)$ & $16(24) * * *$ & 0.709 & 8 & \\
\hline
\end{tabular}

$\underline{\text { Proportion Correct }}$

Control Group

\begin{tabular}{lcccccc} 
Negative & $.972(.026)$ & $.965(.042)$ & $.007(.047)$ & 0.148 & -.010 & .024 \\
Neutral & $.968(.025)$ & $.974(.022)$ & $-.006(.027)$ & 0.206 & -.015 & .004 \\
Positive & $.965(.027)$ & $.975(.033)$ & $-.010(.039)$ & 0.257 & -.024 & .004 \\
Reward Group & & & & & & \\
Negative & $.949(.055)$ & $.942(.058)$ & $.007(.059)$ & 0.128 & -.014 & .029 \\
Neutral & $.951(.045)$ & $.947(.043)$ & $.004(.029)$ & 0.156 & -.006 & .015 \\
Positive & $.945(.040)$ & $.956(.056)$ & $-.011(.048)$ & 0.236 & -.028 & .006 \\
\hline
\end{tabular}

Note: Distraction indices are [distractor-present RT - distractor-absent RT] and [distractor-absent proportion correct - distractor-present proportion correct]. Effect sizes are Cohen's $d_{z}$ from paired comparisons of distractor-absent versus distractor-present trials within conditions. $* * p<.010 . * * * p$ $<.001$ for comparisons of the distraction indices against zero. $95 \%$ confidence intervals surround the distraction scores, in ms and proportion correct. $n=32$ per group. 


\section{Response times}

To examine the influence of reward on task performance, correct RTs were analysed in a 2 (reward group: reward, control) $\times 2$ (distractor presence: distractor-present, distractor-absent) $\times 3$ (distractor valence: negative, neutral, positive) mixed-model ANOVA, with reward as the between-subjects factor and the other factors within-subjects. Participants were $21 \mathrm{~ms}$ slower on distractor-present $(M=522 \mathrm{~ms}, S D=76 \mathrm{~ms})$ than distractor-absent trials $(M=501 \mathrm{~ms}, S D$ $=61 \mathrm{~ms})$, reflected by the main effect of distractor presence, $F(1,62)=55.71, p<.001, \eta_{p}{ }^{2}=$ $.473, \omega^{2}=.431$. Surprisingly, emotional images were not significantly more distracting than neutral ones: the predicted distractor presence $\times$ distractor valence interaction did not reach significance, $F(2,124)=2.55, p=.082, \eta_{p}{ }^{2}=.040, \omega^{2}=.024$. Although the interaction was not significant, we conducted planned comparisons of distraction indices [distractor-present RT - distractor-absent RT] across conditions, which showed that positive images $(M=25 \mathrm{ms,}$ $S D=33 \mathrm{~ms})$ were significantly more distracting than neutral images $(M=16 \mathrm{~ms}, S D=25$ $\mathrm{ms}), t(63)=2.38, p=.020, d_{z}=0.298,95 \%$ CI $[2,17]$. In contrast, negative images $(M=22$ $\mathrm{ms}, S D=34 \mathrm{~ms}$ ) were not significantly more distracting than neutral images, $t(63)=1.48, p$ $=.143, d_{z}=0.185,95 \%$ CI $[-2,15]$, or positive images, $t(63)=0.68, p=.500, d_{z}=0.085$, $95 \%$ CI $[-6,12]$.

Reward sped up responses overall, reflected by the predicted main effect of reward group, $F(1,62)=18.60, p<.001, \eta_{p}{ }^{2}=.231, \omega^{2}=.216($ control group: $M=544 \mathrm{~ms}, S D=65$ ms; reward group: $M=479 \mathrm{~ms}, S D=54 \mathrm{~ms}$ ). Importantly, this main effect was qualified by the predicted reward group $\times$ distractor presence interaction, $F(1,62)=8.11, p=.006, \eta_{p}{ }^{2}=$. $116, \omega^{2}=.056$. Thus, to explore the reward group $\times$ distractor presence interaction, we compared group differences in distraction (collapsed across the three valences). Participants in the control group were significantly more distracted $(M=29 \mathrm{~ms}, S D=26 \mathrm{~ms})$ than those in the reward group $(M=13 \mathrm{~ms}, S D=18 \mathrm{~ms}), t(55.7)=2.85, p=.006, d_{s}=0.712$. The 
absence of a reward group $\times$ distractor presence $\times$ distractor valence interaction, $F(2,124)=$ $0.04, p=.965, \eta_{p}{ }^{2}=.001, \omega^{2}<.001$, shows that reward effects did not depend on valence; contrary to our previous finding with central distractors (Walsh et al., 2018; 2019), we find no evidence that reward reduced emotional distraction to a greater extent than neutral distraction. No other RT effects reached significance.

\section{Accuracy}

Accuracy (proportion correct) was high overall $(M=.959, S D=.034)$. To parallel the RT analysis, accuracy was analysed in a 2 (reward group: reward, control) $\times 2$ (distractor presence: distractor-present, distractor-absent) $\times 3$ (distractor valence: negative, neutral, positive) mixed-model ANOVA. The reward group $(M=.948, S D=.041)$ was significantly less accurate than the control group $(M=.970, S D=.019), F(1,62)=7.13, p=.010, \eta_{p}{ }^{2}=$ $.103, \omega^{2}=.087$. No other main effects or interactions reached significance, including the predicted main effect of distractor presence, $F(1,62)=0.18, p=.676, \eta_{p}^{2}=.003, \omega^{2}<.001$, or the distractor presence $\times$ distractor valence interaction, $F(2,124)=2.82, p=.063, \eta_{p}{ }^{2}=$ $.044, \omega^{2}=.028$.

The contrasting main effect of reward group in the RT and accuracy analyses reflects a speed-accuracy tradeoff. Participants in the reward group were significantly $(65 \mathrm{~ms})$ faster but also significantly (2\%) less accurate than those in the control group. However, reward did not influence distraction as measured in accuracy, reflected by the non-significant reward group $\times$ distractor presence interaction, $F(1,62)=0.25, p=.616, \eta_{p}^{2}=.004, \omega^{2}<.001$, suggesting that a speed-accuracy tradeoff does not account for the benefit of reward on RT distraction. Moreover, in the reward group there was no significant correlation between RT distraction indices and accuracy distraction indices, $r(30)=-.076, p=.679$, which we would expect if the reduction in RT distraction under reward was accounted for by changes in accuracy. 
As predicted by the DMC framework, performance-contingent monetary reward reduced attentional capture by peripheral distractors. We extend previous findings in which motivation enhanced control of distraction by central emotional images (Padmala \& Pessoa, 2014; Padmala et al., 2017; Walsh et al., 2018; 2019), although the reduction in the present experiment was not greater for emotional than for neutral images (as it was in Walsh et al., 2018; 2019). However, we note that the predicted distractor valence $\mathrm{x}$ distractor presence interaction - which would indicate greater attentional capture by emotional than neutral images - was not significant overall $(p=.082)$, and only positive images were more distracting than neutral images $(p=.020)$. This was unexpected, given robust distraction by peripheral emotional images in similar studies (Grimshaw et al., 2018; Murphy et al., 2018; Kranz et al., 2015), and may therefore reflect lower than expected power to detect predicted interactions with valence. We therefore increased sample size in Experiment 2.

\section{Experiment 2}

In Experiment 2, our primary goal was to replicate the effect of reward on attentional capture by emotional distractors in an experiment with greater statistical power. We also extended the research question to ask whether motivation to reduce emotional distraction can be elicited by non-monetary reward. In the lab, motivation is often manipulated with performancecontingent points, which are then translated into monetary reward (Botvinick \& Braver, 2015; Yee \& Braver, 2018). But in real life, motivation can be influenced by all sorts of other factors, including a desire to meet one's own, or others' standards; time pressure; enjoyment; availability of primary reinforcers like sex or food).

A handful of studies have examined the influence of monetary versus non-monetary reward on cognitive control in non-emotional contexts. Dambacher et al. (2011) and Hübner and Schlösser (2010) had participants complete a flanker task in which they earned points for fast and accurate performance. For one group of participants, points represented greater 
monetary earnings; for another group they did not. In both studies, monetary reward led to better accuracy than non-monetary reward, but only when the payoff contingencies emphasised speed to a greater degree than accuracy. In contrast, Shen and Chun (2011) found that non-monetary and monetary reward resulted in similar performance on an attentional task. However, neither of these studies included the necessary no-reward baseline condition to determine how motivating either manipulation was overall. In a recent study, MäkiMarttunen, Hagen, and Espeseth (2019) showed that performance-contingent points - that were not linked to monetary reward - encouraged a shift to proactive control on a response conflict task. Thus, non-monetary reward can elicit proactive control in non-emotional contexts. Here, we determine whether the motivation to ignore emotional distractors can be elicited even without financial gain. We replicated Experiment 1, with reward participants again earning points, achieving levels, and receiving performance feedback, but with no translation of points to monetary reward.

As in Experiment 1, we expected emotional images to be more distracting than neutral images, and for reward to speed up performance. Crucially, if non-monetary reward reduces attentional capture by emotional distractors, we predicted less distraction in the reward group than the control group. We also predicted that, given a larger sample than in Experiment 1, emotional images might capture attention more effectively than neutral images in the absence of reward, and that reward might reduce emotional to a greater extent than neutral distraction, in line with our previous findings (Walsh et al., 2018; 2019). Hypotheses, predictions, and the analysis plan for Experiment 2 were preregistered ${ }^{3}$ on aspredicted.org (https://aspredicted.org/6yk66.pdf).

\footnotetext{
${ }^{3}$ Here, the only deviation from the preregistration is that we do not present two one-sided equivalence tests (Lakens, 2017) examining whether reward similarly influenced positive and negative distraction. The tests were not conclusive in either direction and therefore did not add anything to the findings or conclusions.
} 


\section{Method}

\section{Participants and Sample Size Determination}

One-hundred female first year psychology students at Victoria University of Wellington completed the experiment. Two participants met the preregistered exclusion criteria of achieving less than $75 \%$ accuracy overall, and less than $60 \%$ accuracy on a single block; two participants met the exclusion criterion of displaying distraction $4 S D$ s above the mean in a condition, leaving a total of 96 participants $(M=18.91$, range $18-29$ years $)$, who were randomly assigned to the reward or control groups ( $n=48$ per group). A sensitivity analysis using G*Power (Faul et al., 2007) determined that this sample size had a 0.8 probability of detecting an effect of reward on distraction greater than or equal to $d_{s}=0.578$ (alpha $=.05$, two-tailed), which is comparable to the observed effect size from Experiment $1\left(d_{s}=0.565\right)$.

\section{Materials, Task, and Procedure}

The experiment was run on E Prime 3.0 (Psychology Software Tools, Pittsburgh, PA, USA). The materials, task, and procedure were identical to those of Experiment 1 except that, in the reward group, participants achieved levels for fast and accurate performance, but they did not translate into monetary reward. Instead, all participants - regardless of reward condition received course credit in an introductory psychology course for their participation.

\section{Results and Discussion}

The following predictions and analyses were preregistered unless otherwise noted. Correct RTs and accuracy for neutral distractor blocks did not differ across the positive and negative halves of the experiment (distractor-present and distractor-absent conditions; $p$ 's > .201), so we again collapsed the neutral distractor blocks in analyses. Mean correct RTs, RT distraction indices [distractor-present RT - distractor-absent RT], mean proportion correct scores, and accuracy distraction indices [distractor-absent proportion correct - distractor- 
present proportion correct] for each condition are presented in Table 2. RT distraction indices for each condition are displayed in the bottom panel of Figure 2.

Table 2. Experiment 2: Mean correct RTs (ms), mean accuracy (proportion correct), RT distraction indices [distractor-present RT - distractor-absent RT], and accuracy distraction indices [distractor-absent RT - distractor-present RT] for each distractor condition (negative, neutral, positive, and distractor-absent), are shown separately for control and reward groups. SDs are in brackets.

\begin{tabular}{llllcl}
\hline $\begin{array}{l}\text { Valence } \\
\text { Block }\end{array}$ & $\begin{array}{l}\text { Distractor- } \\
\text { absent }\end{array}$ & $\begin{array}{l}\text { Distractor- } \\
\text { present }\end{array}$ & Distraction & $d_{z}$ & \multicolumn{2}{c}{$95 \%$ CI } \\
Lower & Upper \\
\hline
\end{tabular}

\section{Control Group}

\section{$\underline{\text { Response Times }}$}

\begin{tabular}{lllllll} 
Negative & $553(67)$ & $584(85)$ & $31(40) * * *$ & 0.774 & 19 & 43 \\
Neutral & $557(64)$ & $570(74)$ & $13(29) * *$ & 0.433 & 4 & 21 \\
Positive & $559(60)$ & $606(103)$ & $47(64) * * *$ & 0.747 & 29 & 66 \\
\multicolumn{2}{l}{ Reward Group } & & & & & \\
Negative & $503(68)$ & $514(75)$ & $11(24) * *$ & 0.447 & 4 & 18 \\
Neutral & $502(53)$ & $511(63)$ & $9(19) * *$ & 0.473 & 4 & 14 \\
Positive & $507(65)$ & $518(64)$ & $11(27) * *$ & 0.425 & 4 & 19
\end{tabular}

\section{Control Group}

\section{Proportion Correct}

\begin{tabular}{lcccccc} 
Negative & $.958(.054)$ & $.962(.044)$ & $-.004(.045)$ & 0.098 & -.017 & .009 \\
Neutral & $.961(.038)$ & $.965(.043)$ & $-.004(.037)$ & 0.114 & -.015 & .006 \\
Positive & $.961(.050)$ & $.961(.050)$ & $.000(.038)$ & 0.011 & -.011 & .011 \\
Reward Group & & & & & & \\
Negative & $.930(.051)$ & $.941(.054)$ & $-.011(.052)$ & 0.215 & -.026 & .003 \\
Neutral & $.937(.043)$ & $.935(.047)$ & $.002(.042)$ & 0.037 & -.011 & .014 \\
Positive & $.940(.043)$ & $.934(.068)$ & $.006(.065)$ & 0.089 & -.013 & .025 \\
\hline
\end{tabular}

Note: Distraction indices are [distractor-present RT - distractor-absent RT] and [distractor-absent proportion correct - distractor-present proportion correct]. Effect sizes are Cohen's $d_{z}$ from paired comparisons of distractor-absent versus distractor-present trials within conditions. ${ }^{* *} p<.010 .{ }^{* * *} p$ 
$<.001$ for comparisons of the distraction indices with zero. 95\% confidence intervals surround the distraction scores, in ms and proportion correct. $n=48$ per group.

\section{Response times}

To examine the influence of reward on performance, correct RTs were analysed in a 2 (reward group: reward, control) $\times 2$ (distractor presence: distractor-present, distractor-absent) $\times 3$ (distractor valence: negative, neutral, positive) mixed-model ANOVA, with reward as the between-subjects factor and the other factors within-subjects. Main effects of valence, $F(1.67,156.92)=6.78, p=.003, \eta_{p}^{2}=.067, \omega^{2}=.056$, and distractor presence $F(1,94)=$ $54.42, p<.001, \eta_{p}{ }^{2}=.367, \omega^{2}=.329$, were qualified by the predicted distractor presence by distractor valence interaction, $F(1.59,149.30)=8.92, p<.001, \eta_{p}{ }^{2}=.087, \omega^{2}=.072$, reflecting the fact that participants were more distracted by emotional than neutral images. Comparing distraction indices [distractor-present RT - distractor-absent RT] across valences showed that the interaction was driven by significantly greater distraction by positive images $(M=29 \mathrm{~ms}, S D=52 \mathrm{~ms})$ than neutral images $(M=11 \mathrm{~ms}, S D=24 \mathrm{~ms}), t(95)=3.72, p<$ $.001, d_{z}=0.380,95 \% \mathrm{CI}[9,29] ;$ and greater distraction by negative $(M=21 \mathrm{~ms}, S D=35$ ms) than neutral images, $t(95)=3.18, p=.002, d_{z}=0.325,95 \%$ CI $[4,17]$. Distraction by positive and negative images did not significantly differ, $t(95)=1.63, p=.108, d_{z}=0.166$, 95\% CI $[-2,19]$. Experiment 2 thus shows a robust effect of emotion on attentional capture by peripheral images.

As in Experiment 1, reward sped up responses, reflected in the predicted main effect of reward group, $F(1,94)=21.50, p<.001, \eta_{p}{ }^{2}=.186, \omega^{2}=.176,($ control group $M=571$ ms, $S D=69 \mathrm{~ms}$; reward group $M=509 \mathrm{~ms}, S D=62 \mathrm{~ms}$ ), which was qualified by the predicted reward group $\times$ distractor presence interaction, $F(1,94)=13.17, p<.001, \eta_{p}{ }^{2}=$ $.123, \omega^{2}=.075$. To explore this interaction, distraction indices [distractor-present RT distractor-absent RT] were compared between the control and reward groups. Participants in 
the control group were significantly more distracted $(M=30 \mathrm{~ms}, S D=36 \mathrm{~ms})$ than those in the reward group $(M=10 \mathrm{~ms}, S D=14 \mathrm{~ms}), t(60.5)=3.63, p<.001, d_{s}=0.710,95 \%$ CI $[9$, 31]. Furthermore, the predicted reward group $\times$ distractor presence $\times$ distractor valence interaction, $F(2,188)=6.58, p=.002, \eta_{p}{ }^{2}=.065, \omega^{2}=.051$, was explored by comparing distraction indices between groups, separately for each valence. Negative distraction was reduced in the reward group $(M=11 \mathrm{~ms}, S D=24 \mathrm{~ms})$ relative to the control group $(M=31$ $\mathrm{ms}, S D=40 \mathrm{~ms}), t(77.5)=2.98, p=.004, d_{s}=0.608,95 \%$ CI $[7,34]$; likewise, positive distraction was reduced in the reward group $(M=11 \mathrm{~ms}, S D=27 \mathrm{~ms})$ relative to the control group $(M=48 \mathrm{~ms}, S D=64 \mathrm{~ms}), t(63.2)=3.62, p<.001, d_{s}=0.739,95 \%$ CI $[16,56]$; in contrast, neutral distraction did not differ between the reward $(M=9 \mathrm{~ms}, S D=19 \mathrm{~ms})$ and control $(M=13 \mathrm{~ms}, S D=29 \mathrm{~ms})$ groups, $t(79.6)=0.78, p=.439, d_{s}=0.159,95 \%$ CI $[-6$, 14]. No other RT effects reached significance.

\section{Accuracy}

Accuracy was high overall $(M=.949, S D=.040)$. To parallel the RT analysis, accuracy (proportion correct) was analysed in a 2 (reward group: reward, control) $\times 2$ (distractor presence: present, absent) $\times 3$ (distractor valence: negative, neutral, positive) mixed-model ANOVA. As predicted, the reward group $(M=.936, S D=.038)$ was significantly less accurate than the control group $(M=.961, S D=.038), F(1,94)=10.60, p=.002, \eta_{p}{ }^{2}=.102$, $\omega^{2}=.091$. No other main effects or interactions reached significance, including the main effect of distractor presence, $F(1,94)=0.54, p=.462, \eta_{p}{ }^{2}=.006, \omega^{2}<.001$, nor the distractor presence $\times$ distractor valence interaction, $F(2,188)=1.22, p=.298, \eta_{p}^{2}=.013, \omega^{2}=.002$, both of which were predicted.

As in Experiment 1, we observed a speed-accuracy tradeoff; participants in the reward group were significantly $(62 \mathrm{~ms})$ faster but also significantly $(2.5 \%)$ less accurate than those 
in the control group. However, reward did not influence distraction as measured in accuracy, reflected by the non-significant reward group $\times$ distractor presence interaction, $F(1,94)=$ $0.08, p=.780, \eta_{p}{ }^{2}=.001, \omega^{2}<.001$, suggesting that a speed-accuracy tradeoff does not account for the effect of reward on distraction in RT. Moreover, in the reward group there was no significant correlation between RT distraction indices and accuracy distraction indices, $r(46)=.032, p=.827$, which we would expect if the reduction in RT distraction under reward was accounted for by changes in accuracy.

In our better-powered Experiment 2, we observed greater attentional capture by emotional than neutral images, as expected. Performance-contingent reward reduced this attentional capture, replicating the main finding from Experiment 1 . However, in contrast to Experiment 1, the benefit of reward was specific to emotional distractors. Experiment 2 shows that motivation can be elicited even by non-monetary reward to reduce attentional capture by emotional distractors.

\section{General Discussion}

In these experiments, we show that motivation reduces attentional capture by both positive and negative emotional images, and that the benefits of motivation are not dependent on financial gain. Our findings extend previous work (Padmala \& Pessoa, 2014; Padmala et al., 2017; Walsh et al., 2018; 2019) showing that reward reduces distraction by central emotional images, and contribute to a growing body of evidence showing that attention to emotional stimuli is subject to top-down cognitive control (Grimshaw et al., 2018; Gupta et al., 2016; Okon-Singer et al., 2007; Schmidt et al., 2020).

The specific mechanisms of control that are facilitated by the availability of reward remain to be determined. Our findings are consistent with the DMC framework (Braver, 2012; Braver et al., 2007), which predicts that reward should make the effort of proactive control worthwhile. In non-emotional contexts, the effects of reward on cognitive control 
have been assessed using tasks like the AX-CPT (e.g., Chiew \& Braver 2013; 2014), which allow one to isolate behavioural markers of proactive and reactive control. In these studies, rewards encourage the upregulation of proactive control; by extension we might speculate that rewards similarly encourage proactive control of attentional capture.

However, it is also possible that rewards encourage an upregulation of reactive control, facilitating rapid disengagement from distractors. Even in non-emotional contexts there is debate as to whether attentional capture by salient singleton distractors can be prevented entirely (see Gaspelin \& Luck, 2018; Sawaki \& Luck, 2010); or whether we rapidly disengage from distractors after they capture attention (Belopolsky, Schreij, \& Theeuwes, 2010; Dieciuc, Roque, \& Boot, 2019; Geng, 2014; Theeuwes, 2010; Theeuwes, de Vries, \& Godijn, 2003). Given the evolutionary importance of emotional stimuli, it is plausible that reactive mechanisms might play an even bigger role in controlling emotional than neutral distraction. It is also possible that reward affects proactive and reactive mechanisms in concert. Indeed, pupil dilation findings (Walsh et al., 2019) - which index the timecourse of cognitive control - suggest that reward activates proactive mechanisms (e.g., enhancing goal representation prior to distractor-onset), which then facilitate reactive mechanisms that implement rapid disengagement from emotional distractors and shift attention back to task-relevant stimuli. Future work using time-sensitive electrophysiological (e.g., ERPs) and psychophysiological measures (e.g., pupillometry) will be necessary to elucidate the timecourse of motivation-elicited control of peripheral emotional distractions so as to discriminate between proactive and reactive control mechanisms.

The beneficial effects of rewards did not depend on their monetary value. Few studies have compared the effects of monetary and non-monetary rewards on cognitive control, only in non-emotional contexts (e.g., Dambacher et al., 2011; Hübner \& Schlösser; 2010; MäkiMarttunen et al., 2019; Shen \& Chun, 2011), and yielding mixed results. Inconsistencies 
across studies may reflect differences in the specific reward manipulations that were used. In their recent theoretical review, Notebaert and Braem (2016) suggest that different components of reward may have dissociable influences on cognitive control. Specifically, motivation is proposed to encourage anticipation and therefore proactive control, whereas positive affect is proposed to encourage environmental exploration, and therefore reactive control. To maximise the impact of our reward manipulation we used a combination of affective (pleasant sounds, words of encouragement, coin animations) and motivational (points, levels, feedback) components, and so we cannot identify the specific component(s) that affected cognitive control. Determining the additive and individual influence of different reward components in future studies may well inform theoretical understanding of how rewards affect cognitive control. For example, work directly comparing the influence of performance-contingent versus non-contingent reward, or affectively neutral versus affectively positive feedback, would help to disentangle the respective motivational and affective influences of reward on control.

Beyond these theoretical implications, our work has practical implications both inside and outside the lab. First, the fact that non-monetary rewards can influence cognitive control means that many types of motivational research can be conducted with unpaid research volunteers. Monetary rewards are the norm in this area, and they place a substantial, but perhaps unnecessary, burden on research budgets. Second, many of our reward components feedback, encouragement, "gamification" - are part of individual laboratory practices that are sometimes not reported in method sections (Brenninkmeijer, Derksen, \& Rietzschel, 2019). The fact that some of these components affect motivation (and thus cognitive control) means that they should be carefully considered and more systematically reported. Finally, our findings also highlight the potential impact non-monetary reward might have on performance outside of the lab (e.g., in the workplace, playing sports, while driving). 
In comparing the results of our two studies, it should be noted that in Experiment 1, participants were recruited from the community and were paid for their time (even those in the non-rewarded condition); in Experiment 2, participants were recruited from the introductory psychology pool and were compensated with course credit. These different reasons for participating might influence task motivation and cognitive control. However, contrary to the notion that student samples lack motivation (Krug \& Braver, 2014), participants in the control conditions of the two experiments (which were methodologically identical) showed similar levels of distraction overall (Experiment $1, M=29 \mathrm{~ms}, S D=26 \mathrm{~ms}$ of distraction; Experiment 2, $M=30 \mathrm{~ms}, S D=36 \mathrm{~ms}$ ), suggesting that their potentially different motivations for participation did not influence baseline levels of cognitive control. Most importantly, despite these potential differences, reward reduced attentional capture by emotional images in both studies.

The studies did show different effects of reward on attentional capture by neutral images though. Monetary reward (in Experiment 1) reduced emotional and neutral distraction, whereas non-monetary reward (in Experiment 2) reduced emotional but not neutral distraction. We think it would be premature, however, to attribute this difference to the nature of the rewards. Given that reward reduced emotional to a greater degree than neutral distraction in both our previous studies with central distractors (Walsh et al., 2018; 2019), it is possible that Experiment 1 (with $n=32$ per group) simply lacked power to detect a three-way reward group $\times$ distractor presence $\times$ distractor valence interaction. It might seem curious that reward can reduce emotional to a greater extent than neutral distraction, given that emotional stimuli are harder to ignore. One possibility is that there is more room for improvement because emotional distraction is greater than neutral distraction in the control group. Alternatively, reward may influence attention to emotional versus neutral stimuli differently. For example, reward might enhance the goal-relevance of the target stimuli, 
allowing them to better compete for goal-driven attention with the emotional distractors. Future work using blocked versus intermixed distractor valence designs (i.e., when people can anticipate distractor valence, or not) may shed light on this hypothesis. Emotional distractors are always relevant to the fundamental, biologically-determined goals of surviving and reproducing. Because attention to neutral images is less driven by these goals, their ability to capture attention may not be influenced by such changes in motivational value.

Research was supported by a grant from the Royal Society of New Zealand Marsden Fund (VUW1307) to Gina Grimshaw and David Carmel.

The authors report no conflict of interest.

\section{References}

Azzopardi, P., \& Cowey, A. (1993). Preferential representation of the fovea in the primary visual cortex. Nature, 361(6414), 719-721. https://doi.org/10.1038/361719a0

Beck, D. M., \& Lavie, N. (2005). Look here but ignore what you see: Effects of distractors at fixation. Journal of Experimental Psychology: Human Perception and Performance, 31(3), 592-607. https://doi.org/10.1037/0096-1523.31.3.592

Belopolsky, A. V., Schreij, D., \& Theeuwes, J. (2010). What is top-down about contingent capture? Attention, Perception, \& Psychophysics, 72(2), 326-341. https://doi.org/10.3758/APP.72.2.326

Berardi, N., \& Fiorentini, A. (1991). Visual field asymmetries in pattern discrimination: A sign of asymmetry in cortical visual field representation? Vision Research, 31(10), 1831-1836. https://doi.org/10.1016/0042-6989(91)90030-9 
Botvinick, M., \& Braver, T. (2015). Motivation and cognitive control: From behavior to neural mechanism. Annual Review of Psychology, 66(1), 83-113. https://doi.org/10.1146/annurev-psych-010814-015044

Braver, T. S. (2012). The variable nature of cognitive control: A dual mechanisms framework. Trends in Cognitive Sciences, 16(2), 106-113. https://doi.org/10.1016/j.tics.2011.12.010

Braver, T. S., Gray, J. R., \& Burgess, G. C. (2007). Explaining the many varieties of working memory variation: Dual mechanisms of cognitive control. In Variation in working memory (pp. 76-106). New York, NY, US: Oxford University Press.

Brenninkmeijer, J., Derksen, M., \& Rietzschel, E. (2019). Informal laboratory practices in psychology. Collabra: Psychology, 5(1), 45. http://doi.org/10.1525/collabra.221

Brown, C. R. H., Berggren, N., \& Forster, S. (2019). Testing a goal-driven account of involuntary attentional capture by threat. Emotion (Washington, D.C.). https://doi.org/10/ggdbx3

Carrasco, M., McElree, B., Denisova, K., \& Giordano, A. M. (2003). Speed of visual processing increases with eccentricity. Nature Neuroscience, 6(7), 699-700. https://doi.org/10.1038/nn1079

Carretié, L. (2014). Exogenous (automatic) attention to emotional stimuli: A review. Cognitive, Affective, \& Behavioral Neuroscience, 14(4), 1228-1258. https://doi.org/10.3758/s13415-014-0270-2

Carretié, L., Albert, J., López-Martín, S., Hoyos, S., Kessel, D., Tapia, M., \& Capilla, A. (2013). Differential neural mechanisms underlying exogenous attention to peripheral and central distracters. Neuropsychologia, 51(10), 1838-1847. https://doi.org/10.1016/j.neuropsychologia.2013.06.021 
Carver, C. S., \& White, T. L. (1994). Behavioral inhibition, behavioral activation, and affective responses to impending reward and punishment: The BIS/BAS scales. Journal of Personality and Social Psychology, 67(2), 319-333.

Chiew, K. S., \& Braver, T. S. (2014). Dissociable influences of reward motivation and positive emotion on cognitive control. Cognitive, Affective, \& Behavioral Neuroscience, 14(2), 509-529. https://doi.org/10.3758/s13415-014-0280-0

Cohen, J. (1992). A power primer. Psychological Bulletin, 112(1), 155-159.

Cohen, J. D. (2017). Cognitive control. In The Wiley Handbook of Cognitive Control (pp. 128). https://doi.org/10.1002/9781118920497.ch1

Corbetta, M., Akbudak, E., Conturo, T. E., Snyder, A. Z., Ollinger, J. M., Drury, H. A., ... Shulman, G. L. (1998). A common network of functional areas for attention and eye movements. Neuron, 21(4), 761-773. https://doi.org/10.1016/S0896-6273(00)805930

Dambacher, M., Hübner, R., \& Schlösser, J. (2011). Monetary incentives in speeded perceptual decision: Effects of penalizing errors versus slow responses. Frontiers in Psychology, 2. https://doi.org/10.3389/fpsyg.2011.00248

Delacre, M., Lakens, D., \& Leys, C. (2017). Why psychologists should by default use Welch's $t$-test instead of Student's $t$-test. International Review of Social Psychology, 30(1), 92-101. https://doi.org/10.5334/irsp.82

Desimone, R., \& Duncan, J. (1995). Neural mechanisms of selective visual attention. Annual Review of Neuroscience, 18(1), 193-222. https://doi.org/10.1146/annurev.ne.18.030195.001205

Dieciuc, M. A., Roque, N. A., \& Boot, W. R. (2019). The spatial dynamics of mouse-tracking reveal that attention capture is stimulus-driven rather than contingent upon top-down 
goals. Journal of Experimental Psychology: Human Perception and Performance, 45(10), 1285-1290. https://doi.org/10.1037/xhp0000671

Dolcos, F., Katsumi, Y., Moore, M., Berggren, N., de Gelder, B., Derakshan, N., ... Dolcos, S. (2019). Neural correlates of emotion-attention interactions: From perception, learning and memory to individual differences and training interventions. Neuroscience \& Biobehavioral Reviews. https://doi.org/10.1016/j.neubiorev.2019.08.017

Etzel, J. A., Cole, M. W., Zacks, J. M., Kay, K. N., \& Braver, T. S. (2016). Reward motivation enhances task coding in frontoparietal cortex. Cerebral Cortex, 26(4), 1647-1659. https://doi.org/10.1093/cercor/bhu327

Faul, F., Erdfelder, E., Lang, A.-G., \& Buchner, A. (2007). G*Power 3: A flexible statistical power analysis program for the social, behavioral, and biomedical sciences. Behavior Research Methods, 39(2), 175-191. https://doi.org/10.3758/BF03193146

Forster, S., \& Lavie, N. (2008). Attentional capture by entirely irrelevant distractors. Visual Cognition, 16(2-3), 200-214. https://doi.org/10.1080/13506280701465049

Gaspelin, N., \& Luck, S. J. (2018). Combined electrophysiological and behavioral evidence for the suppression of salient distractors. Journal of Cognitive Neuroscience, 1-16. https://doi.org/10.1162/jocn_a_01279

Geng, J. J. (2014). Attentional mechanisms of distractor suppression. Current Directions in Psychological Science, 23(2), 147-153. https://doi.org/10.1177/0963721414525780

Gratton, G., Cooper, P., Fabiani, M., Carter, C. S., \& Karayanidis, F. (2018). Dynamics of cognitive control: Theoretical bases, paradigms, and a view for the future. Psychophysiology, 55(3), e13016. https://doi.org/10.1111/psyp.13016 
Grimshaw, G. M., Kranz, L. S., Carmel, D., Moody, R. E., \& Devue, C. (2018). Contrasting reactive and proactive control of emotional distraction. Emotion, 18(1), 26-38. https://doi.org/10.1037/emo0000337

Gupta, R., Hur, Y., \& Lavie, N. (2016). Distracted by pleasure: Effects of positive versus negative valence on emotional capture under load. Emotion, 16(3), 328-337. https://doi.org/10.1037/emo0000112

Hübner, R., \& Schlösser, J. (2010). Monetary reward increases attentional effort in the flanker task. Psychonomic Bulletin \& Review, 17(6), 821-826. https://doi.org/10.3758/PBR.17.6.821

Kool, W., Shenhav, A., \& Botvinick, M. M. (2017). Cognitive control as cost-benefit decision making. In The Wiley Handbook of Cognitive Control (pp. 167-189). https://doi.org/10.1002/9781118920497.ch10

Krug, M. K., \& Braver, T. S. (2014). Motivation and cognitive control: Going beyond monetary incentives. In E. Bijleveld \& H. Aarts (Eds.), The Psychological Science of Money (pp. 137-162). https://doi.org/10.1007/978-1-4939-0959-9_7

Lakens, D. (2013). Calculating and reporting effect sizes to facilitate cumulative science: A practical primer for t-tests and ANOVAs. Frontiers in Psychology, 4. https://doi.org/10.3389/fpsyg.2013.00863

Lang, P. J., \& Bradley, M. M. (2010). Emotion and the motivational brain. Biological Psychology, 84(3), 437-450. https://doi.org/10.1016/j.biopsycho.2009.10.007

Mäki-Marttunen, V., Hagen, T., \& Espeseth, T. (2019). Proactive and reactive modes of cognitive control can operate independently and simultaneously. Acta Psychologica, 199, 102891. https://doi.org/10.1016/j.actpsy.2019.102891

LeDoux, J. (2012). Rethinking the emotional brain. Neuron, 73(4), 653-676. https://doi.org/10.1016/j.neuron.2012.02.004 
Miller, E. K. (2000). The prefontral cortex and cognitive control. Nature Reviews Neuroscience, 1(1), 59-65. https://doi.org/10.1038/35036228

Mohanty, A., \& Sussman, T. J. (2013). Top-down modulation of attention by emotion. Frontiers in Human Neuroscience, 7. https://doi.org/10.3389/fnhum.2013.00102

Most, S. B., Chun, M. M., Widders, D. M., \& Zald, D. H. (2005). Attentional rubbernecking: Cognitive control and personality in emotion-induced blindness. Psychonomic Bulletin \& Review, 12(4), 654-661. https://doi.org/10.3758/BF03196754

Mulckhuyse, M. (2018). The influence of emotional stimuli on the oculomotor system: A review of the literature. Cognitive, Affective, \& Behavioral Neuroscience. https://doi.org/10.3758/s13415-018-0590-8

Öhman, A., Flykt, A., \& Esteves, F. (2001). Emotion drives attention: Detecting the snake in the grass. Journal of Experimental Psychology. General, 130(3), 466-478. https://doi.org/10/b6fthv

Okon-Singer, H., Lichtenstein-Vidne, L., \& Cohen, N. (2013). Dynamic modulation of emotional processing. Biological Psychology, 92(3), 480-491. https://doi.org/10.1016/j.biopsycho.2012.05.010

Okon-Singer, H., Tzelgov, J., \& Henik, A. (2007). Distinguishing between automaticity and attention in the processing of emotionally significant stimuli. Emotion, 7(1), 147-157. https://doi.org/10.1037/1528-3542.7.1.147

Padmala, S., \& Pessoa, L. (2011). Reward reduces conflict by enhancing attentional control and biasing visual cortical processing. Journal of Cognitive Neuroscience, 23(11), 3419-3432. https://doi.org/10.1162/jocn_a_00011

Padmala, S., \& Pessoa, L. (2014). Motivation versus aversive processing during perception. Emotion, 14(3), 450-454. https://doi.org/10.1037/a0036112 
Padmala, S., Sirbu, M., \& Pessoa, L. (2017). Potential reward reduces the adverse impact of negative distractor stimuli. Social Cognitive and Affective Neuroscience, 12(9), 14021413. https://doi.org/10.1093/scan/nsx067

Posner, M. I. (1980). Orienting of attention. Quarterly Journal of Experimental Psychology, 32(1), 3-25. https://doi.org/10.1080/00335558008248231

Pourtois, G., Schettino, A., \& Vuilleumier, P. (2013). Brain mechanisms for emotional influences on perception and attention: What is magic and what is not. Biological Psychology, 92(3), 492-512. https://doi.org/10.1016/j.biopsycho.2012.02.007

Rolls, E. T. (2000). On the brain and emotion. Behavioral and Brain Sciences, 23(2), 219228. https://doi.org/10.1017/S0140525X00512424

RStudio Team (2019). RStudio: Integrated Development for R. RStudio, Inc., Boston, MA URL http://www.rstudio.com/.

Sawaki, R., \& Luck, S. J. (2010). Capture versus suppression of attention by salient singletons: Electrophysiological evidence for an automatic attend-to-me signal. Attention, Perception, \& Psychophysics, 72(6), 1455-1470. https://doi.org/10.3758/APP.72.6.1455

Shen, Y. J., \& Chun, M. M. (2011). Increases in rewards promote flexible behavior. Attention, Perception, \& Psychophysics, 73(3), 938-952. https://doi.org/10.3758/s13414-010-0065-7

Shenhav, A. (2017). The perils of losing control: Why self-control is not just another valuebased decision. Psychological Inquiry, 28(2-3), 148-152. https://doi.org/10.1080/1047840X.2017.1337407

Schmidts, C., Foerster, A., Kleinsorge, T., \& Kunde, W. (2020). Proactive control of affective distraction: Experience-based but not expectancy-based. Cognition, 194, 104072. https://doi.org/10.1016/j.cognition.2019.104072 
The jamovi project (2019). jamovi (Version 0.9) [Computer Software]. Retrieved from https://www.jamovi.org

Theeuwes, J. (1994). Stimulus-driven capture and attentional set: Selective search for color and visual abrupt onsets. Journal of Experimental Psychology: Human Perception and Performance, 20(4), 799-806.

Theeuwes, J. (1991). Exogenous and endogenous control of attention: The effect of visual onsets and offsets. Perception \& Psychophysics, 49(1), 83-90. https://doi.org/10.3758/BF03211619

Theeuwes, J. (2010). Top-down and bottom-up control of visual selection. Acta Psychologica, 135(2), 77-99. https://doi.org/10.1016/j.actpsy.2010.02.006

Theeuwes, J, de Vries, G.-J., \& Godijn, R. (2003). Attentional and oculomotor capture with static singletons. Perception \& Psychophysics, 65(5), 735-746. https://doi.org/10.3758/BF03194810

van den Berg, B., Krebs, R. M., Lorist, M. M., \& Woldorff, M. G. (2014). Utilization of reward-prospect enhances preparatory attention and reduces stimulus conflict. Cognitive, Affective \& Behavioral Neuroscience, 14(2), 561-577. https://doi.org/10.3758/s13415-014-0281-z

Vuilleumier, P. (2015). Affective and motivational control of vision. Current Opinion in Neurology, 28(1), 29. https://doi.org/10.1097/WCO.0000000000000159

Walker, R., Walker, D. G., Husain, M., \& Kennard, C. (2000). Control of voluntary and reflexive saccades. Experimental Brain Research, 130(4), 540-544. https://doi.org/10/bdrpjj

Walsh, A. T., Carmel, D., \& Grimshaw, G. M. (2019). Reward elicits cognitive control over emotional distraction: Evidence from pupillometry. Cognitive, Affective, \& 
Behavioral Neuroscience, 19(3), 537-554. https://doi.org/10.3758/s13415-01800669-w

Walsh, A. T., Carmel, D., Harper, D., \& Grimshaw, G. M. (2018). Motivation enhances control of positive and negative emotional distractions. Psychonomic Bulletin \& Review, 25(4), 1556-1562. https://doi.org/10.3758/s13423-017-1414-5

Wickham, H. ggplot2: Elegant Graphics for Data Analysis. Springer-Verlag New York, 2016.

Willenbockel, V., Sadr, J., Fiset, D., Horne, G. O., Gosselin, F., \& Tanaka, J. W. (2010). Controlling low-level image properties: The SHINE toolbox. Behavior Research Methods, 42(3), 671-684. https://doi.org/10.3758/BRM.42.3.671

Yee, D. M., \& Braver, T. S. (2018). Interactions of motivation and cognitive control. Current Opinion in Behavioral Sciences, 19, 83-90.

https://doi.org/10.1016/j.cobeha.2017.11.009

Yiend, J. (2010). The effects of emotion on attention: A review of attentional processing of emotional information. Cognition and Emotion, 24(1), 3-47.

https://doi.org/10.1080/02699930903205698 


\section{Supplementary Analyses: Proportional Distraction Indices}

\section{Experiment 1.}

To check whether the overall group RT difference systematically affected our key findings, we conducted a mixed-model ANOVA on proportional distraction indices [(distractor-present RT - distractor-absent RT / distractor-absent RT) * 100], with reward group (reward, controls) as the between-subjects factor and distractor valence (negative, neutral, positive) as the within-subjects factor. There was a significant effect of reward on proportional distraction indices, $F(1,62)=6.75, p=.012, \eta_{p}{ }^{2}=.098$ (control group: $M=5.23 \%, S D=4.36 \%$; reward group: $M=2.63 \%, S D=3.59 \%$ ). Thus, even when taking the overall RT difference into account, the reward group was less distracted than the control group. Consistent with the RT findings, neither the distractor valence main effect, nor the distractor valence $\mathrm{x}$ reward group interaction were significant (both $p$ 's $>.187$ ).

\section{Experiment 2.}

The same mixed-model ANOVA was run on proportional distraction indices [(distractorpresent RT - distractor-absent RT / distractor-absent RT) * 100] for Experiment 2. As for Experiment 1, there was a significant effect of reward on proportional distraction indices, $F(1,94)=12.10, p<.001, \eta_{p}{ }^{2}=.114($ control group: $M=5.31 \%, S D=5.95 \%$; reward group: $M=2.03 \%, S D=2.65 \%)$. In Experiment 2, consistent with the RT analyses, there was also a significant main effect of distractor valence, $F(1.57,147.95)=9.87, p<.001, \eta_{p}{ }^{2}$ $=.095$ (i.e., the level of distraction depended on the valence of the distractor). These two main effects were qualified by a significant reward group x distractor valence interaction, $F(2,188)=3.97, p=.020, \eta_{p}{ }^{2}=.041$, which was further explored with independent-sample $t$ tests, comparing proportional distraction indices, for each valence, between the reward and control groups. Consistent with findings from the main RT analyses, negative distraction was reduced in the reward group $(M=1.77 \%, S D=4.86 \%)$ relative to the control group $(M=$ 
$4.74 \%, S D=7.36 \%), t(81.5)=2.34, p=.022, d_{s}=0.477,95 \%$ CI $[0.44,5.51]$; likewise, positive distraction was reduced in the reward group $(M=2.87 \%, S D=5.01 \%)$ relative to the control group $(M=8.82 \%, S D=11.82 \%), t(63.3)=3.21, p=.002, d_{s}=0.655,95 \%$ CI $[2.24$, 9.65]; in contrast, neutral distraction did not differ between the reward $(M=1.46 \%, S D=$ $3.27 \%)$ and control $(M=2.18 \%, S D=5.39 \%)$ groups, $t(76.8)=0.97, p=.335, d_{s}=0.198$, 95\% CI $[-0.94,2.73]$. Thus, even when factoring the overall group difference in RTs into these proportional distraction indices, reward reduced emotional but not neutral distraction. 